\title{
Primary writing tremor
}

\author{
J . C. ROTHW E L L, M. M. TR A U B, A N D C. D. MAR S D N
}

From the University Department of Neurology, Institute of Psychiatry and King's College Hospital Medical School, London

S UMMARY We describe a patient who complained of jerking of the right forearm on writing. Active pronation of his arm produced several beats of pronation/supination tremor. A burst of tremor also could be elicited by tendon taps to the volar surface of the wrist, to the finger extensors, and to pectoralis major, and by forcible supination of the wrist delivered by a torque motor. The subject's writing difficulty and tremor were temporarily abolished by partial motor point anaesthesia of pronator teres. We conclude that the tremor was caused by an abnormal response to muscle spindle input from pronator teres.

Writing difficulties caused by various mechanisms cccur in a number of neurological diseases (Marsden and Parkes, 1973). Here we give an account of a patient whose main complaint was of progressive inability to write or draw because of sudden bursts of tremor when he firmly pronated his right forearm.

\section{Case report}

At the age of 9 years the patient (AS, Maudsley Hospital No. 760482) suffered meningitis. He was drowsy with a stiff neck and positive Kernig's sign, but had no other neurological deficit. The CSF was purulent but no organisms were cultured or seen on microscopy. He made a full recovery after treatment with antibiotics. Three years later when quite well he suddenly developed shaking and jerking movements of his right hand while wielding a pen, or of either hand when using a knife or holding a cup. After two weeks the abnormal movements of his left hand resolved but his writing and drawing difficulties increased over the next seven years. When he tried to write, the pen would suddenly jerk off the page and shake briefly. A similar short-lived burst of tremor occurred on any motor act involving pronation of the right forearm, as for example, lifting a cup to his lips or using a screwdriver. As a result he had to give up his job as a trainee architectural

Address for reprint requests: Professor C. D. Marsden, University Department of Neurology, Institute of Psychiatry, De Crespigny Park, London SE5 8AZ.

Accepted 19 May 1979 draughtsman, and found ordinary writing and feeding difficult. Three pints of beer improved these abnormal movements markedly until the age of 18 years, but subsequently alcohol was lesso

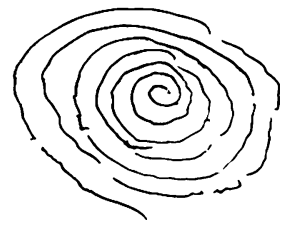

(a)

(b)
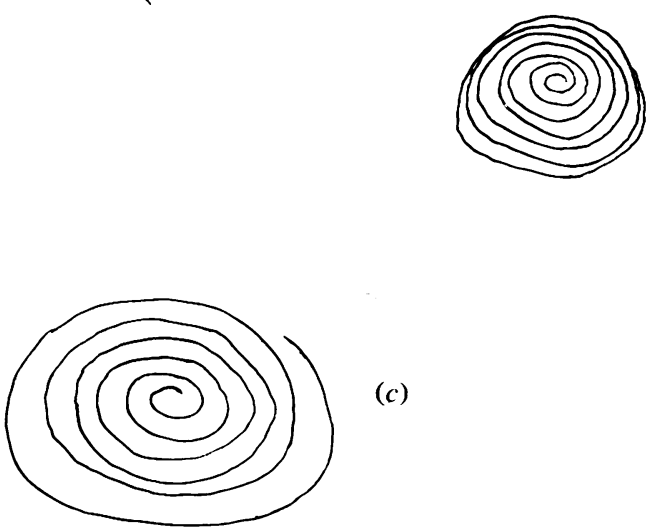

(c)

Fig. 1 Archimedes spirals drawn with the affected right hand. (a) As usual. Note the interruptions to the continuity of the spiral trace, each one of which represents a jerk lifting the pen from the paper. Note also the otherwise accurate drawing. (b) With right wrist held by the patient to prevent pronation/ supination movements. The spiral is now complete. (c) As in (a) after motor point block of pronator teres with $1 \%$ lignocaine (see Methods). 
beneficial. There was no family history of neurological disease or consanguinity.

Examination at the age of 20 years, when he was fully investigated, revealed that his abnormal movements on writing consisted of three to four bursts of pronation/supination tremor of his right forearm when the pen was pushed against the paper (Fig. 1a). However, if the patient drew or wrote with his wrist held rigid and only made movements at his shoulder and elbow no tremor was elicited (Fig. 1b). When the patient held his right arm semipronated bursts of tremor could be evoked by taps with a tendon hammer to the ipsilateral pectoralis major, to the finger extensors near the lateral epicondyle of the humerus and, most effective of all, to the volar surface of the wrist over the head of the radius such as to supinate the forearm. Tendon taps to biceps, triceps, and brachioradialis evoked normal tendon jerks with no subsequent tremor. There was no resting tremor of the right arm, nor was there any tremor of the outstretched arms or with the fingers held in front of the nose. Tests of coordination of the right arm, such as finger-to-nose and rapid movements of individual fingers, were executed normally provided the patient avoided pronation or supination.

A routine electroencephalogram (EEG) showed alpha rhythm at about $11 \mathrm{~Hz}$ bilaterally without asymmetry, but there were slower components and some additional slow or sharp waves were prominent in the left more than the right parietal area (Dr M. Driver). An EMI CAT scan was normal (Dr Hoare), as were routine electromyography (EMG), nerve conduction studies, and somatosensory evoked potentials to median nerve stimulation. There was no abnormality of the cerebrospinal fluid and serology was negative.

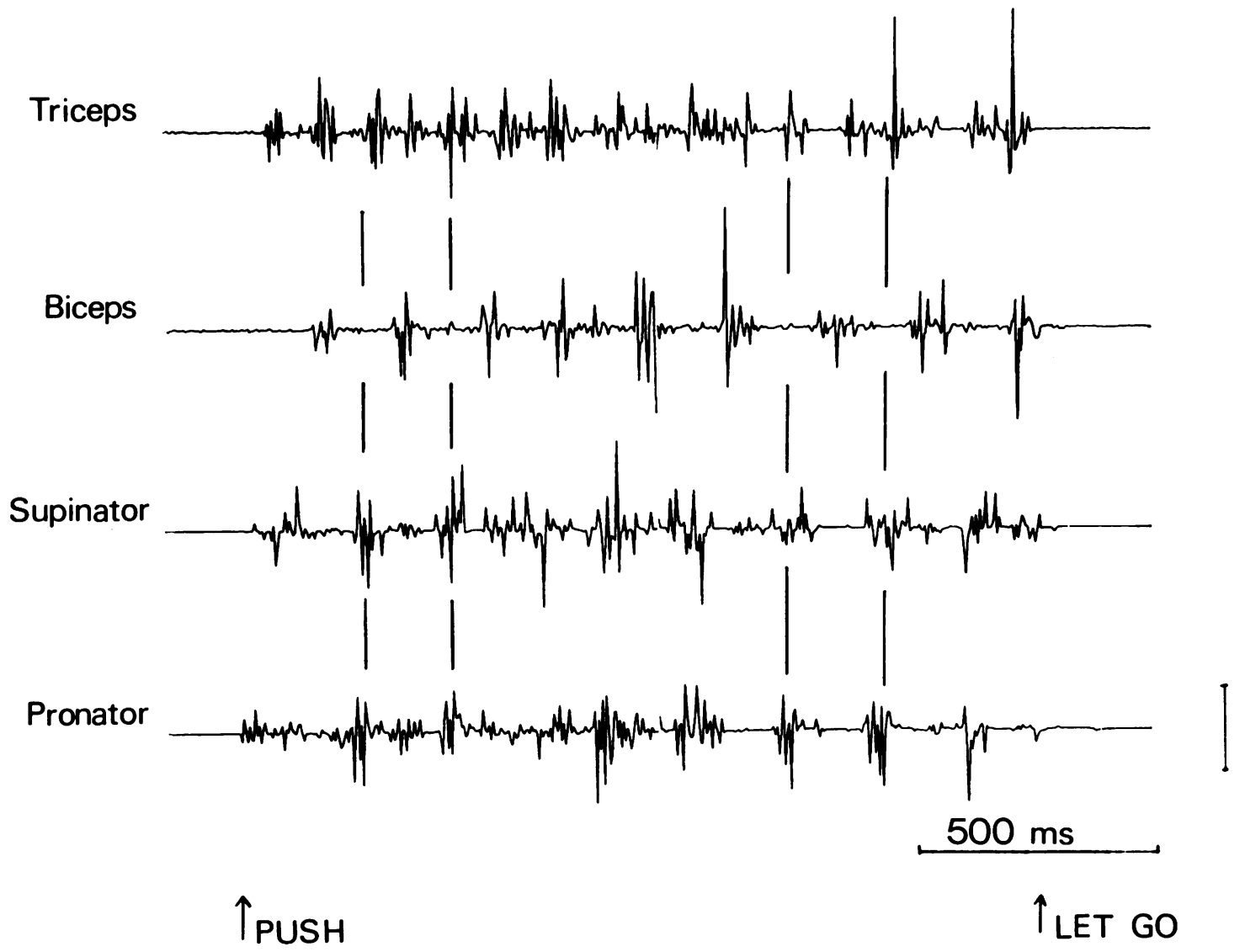

Fig. 2 Typical record of EMG activity from right triceps, biceps, supinator, and pronator teres when the patient pronated the forearm against resistance. Timing bars are shown to emphasise the relation of bursts in different muscles to each other. Vertical calibration: $1 \mathrm{mV}$ for supinator, $400 \mu \mathrm{V}$ for the other muscles. 
Serum copper and caeruloplasmin concentrations were normal.

\section{Methods}

The EMG was recorded using silver/silver-chloride surface electrodes from biceps, triceps, pronator teres, and supinator (the latter inevitably was contaminated by activity in the adjacent and superficial mass of wrist and finger extensors). The EEG was recorded by unipolar electrodes 70 and $110 \mathrm{~mm}$ lateral to the vertex over the arm area of the sensorimotor cortex, referred to an electrode on the nose. Both EMG and EEG signals were preamplified (Devices 3160 amplifiers) with time constants of 0.002 and $1.0 \mathrm{~s}$ respectively $3 \mathrm{~dB}$ down at $2.5 \mathrm{kHz}$. During episodes of right arm jerking elicited by active pronation against resistance the muscle EMG activity was recorded by a PDP 12 computer, using a continuous collecting programme (ADCON, JCR). Alterna- tively, tremor was elicited by suddenly pronating the right forearm with a pulse of force delivered by a torque motor (Printed Motors G9M4). The patient grasped a T-piece attached to the motor spindle and held his semipronated forearm horizontal in line with the axis of rotation of the motor. He attempted to maintain the T-piece in a constant position by pushing against the force offered by the motor. Every 8-10 seconds the motor torque was increased from a background level of $1.2 \mathrm{Nm}$ to $2.4 \mathrm{Nm}$ for $1.5 \mathrm{~s}$ to stretch pronator teres. This elicited a short burst of pronation/supination tremor. Jerks also were evoked by the experimenter delivering a blow with a tendon hammer to pectoralis major or to the wrist as described above. Electromyographic data were collected by the computer which was triggered by the delivery of the stimulus in single trials or in averages of up to 32 trials (using the programme AV, Mr H. B. Morton). To investigate the relationship between EMG and EEG events,

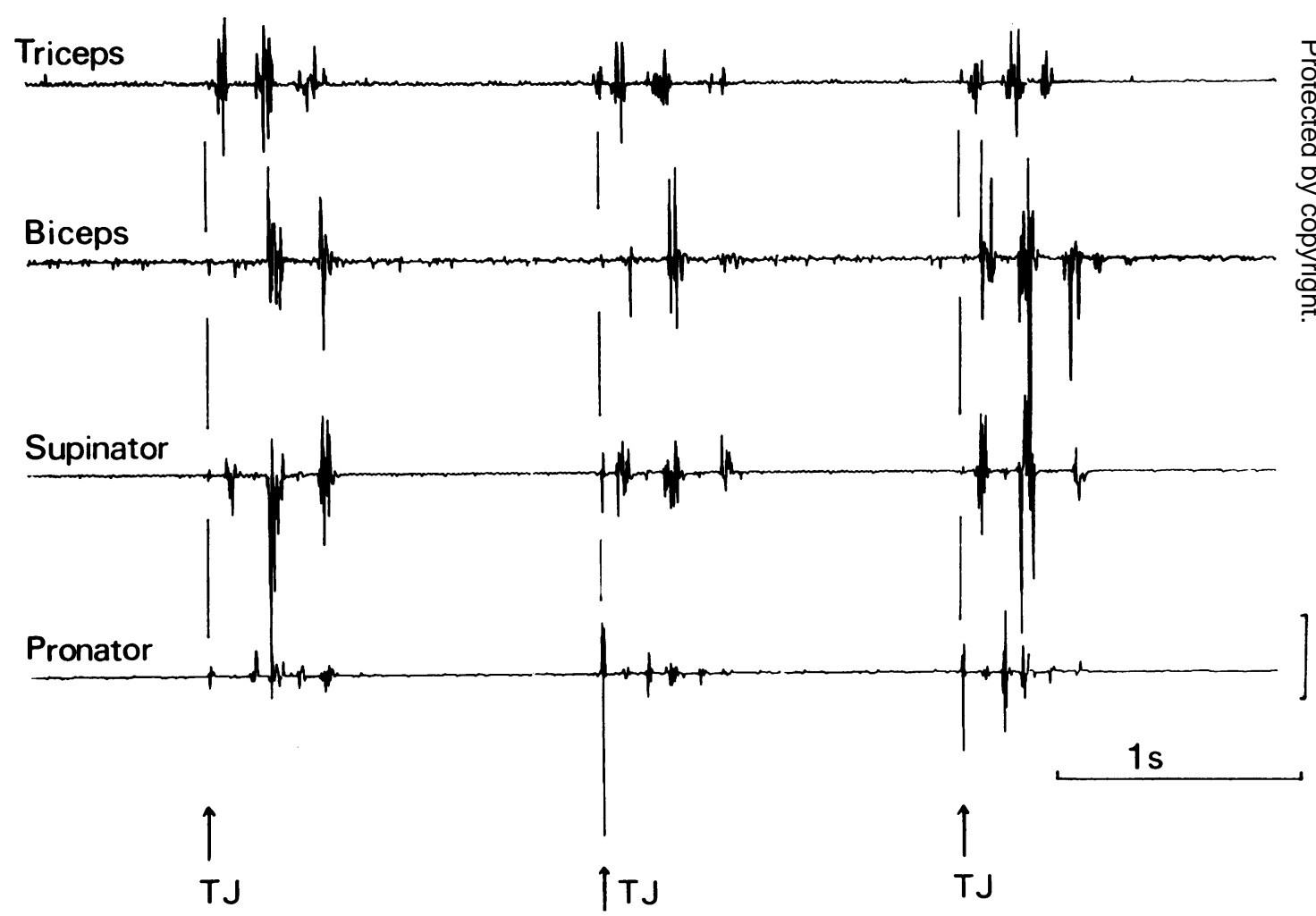

Fig. 3 EMG response of right triceps, biceps, supinator, and pronator teres to three taps with a tendon hammer to the volar surface of the wrist, such as to supinate the forearm. Timing bars coincident with the monosynaptic tendon jerk (arrow-TJ) in pronator teres are shown. Vertical calibration: $1 \mathrm{mV}$ for supinator, $400 \mu \mathrm{V}$ for other muscles. 
jerks were averaged using the programme PASTIME (Mr H. B. Morton) triggering the computer from the EMG of one of the active muscles in order to observe EEG events before and after the trigger.

In some experiments $3 \mathrm{ml}$ of $1 \%$ lignocaine without adrenaline were injected into the motor point of pronator teres, as identified by previous surface electrical stimulation.

\section{Results}

EMG STUDIES

Figure 2 shows EMG records when the patient pronated the right forearm against resistance. Bursts of polyphasic EMG activity lasting 50$100 \mathrm{~ms}$ occurred at about $4-6 \mathrm{~Hz}$ in biceps, supinator, and pronator teres, while triceps had a tendency to fire at a faster frequency-about double that in other muscles. Supinator and pronator usually contracted simultaneously while biceps contracted between their bursts. This EMG pattern accompanied a rhythmic pronation/ supination tremor, which often continued as long as the subject attempted to exert a steady moderate to strong force. Figure 3 shows the effect of striking the wrist with a tendon hammer such as to stretch the pronator (as described in Methods), which produced an initial monophasic or biphasic tendon jerk (arrowed) most evident in pronator teres, followed by two to three bursts of polyphasic EMG activity at about $6 \mathrm{~Hz}$ responsible for the visible tremor. The EMG bursts were more or less synchronous in all muscles but pronator teres fired at about double the frequency of the others. The latency from the tendon tap to the beginning of the tendon jerk in pronator teres was constant at $17 \mathrm{~ms}$ but that to the beginning of the first EMG burst responsible for the repetitive tremor varied from 128 to $232 \mathrm{~ms}$ (mean $151 \pm 5 \mathrm{SEM}, \mathrm{N}=31$ ) (Fig. 4). This record also shows that each of the latter EMG bursts consists of a series of repetitive discharges in contrast to the single action potential of the tendon jerk. When pronator teres was stretched by the torque motor, the forearm went into oscillation as shown in the position trace in Fig. 5. Electromyographic activity in pronator teres alternated with that in biceps and supinator. The stretch caused a small early tendon jerk in pronator teres, followed by a series of tremor bursts of activity in the muscle, the first of which began $110-120 \mathrm{~ms}$ after the stimulus. The first burst of activity in biceps and supinator began at about 55 and $60 \mathrm{~ms}$ after the stretch, at a time when the supination movement was unloading both muscles.

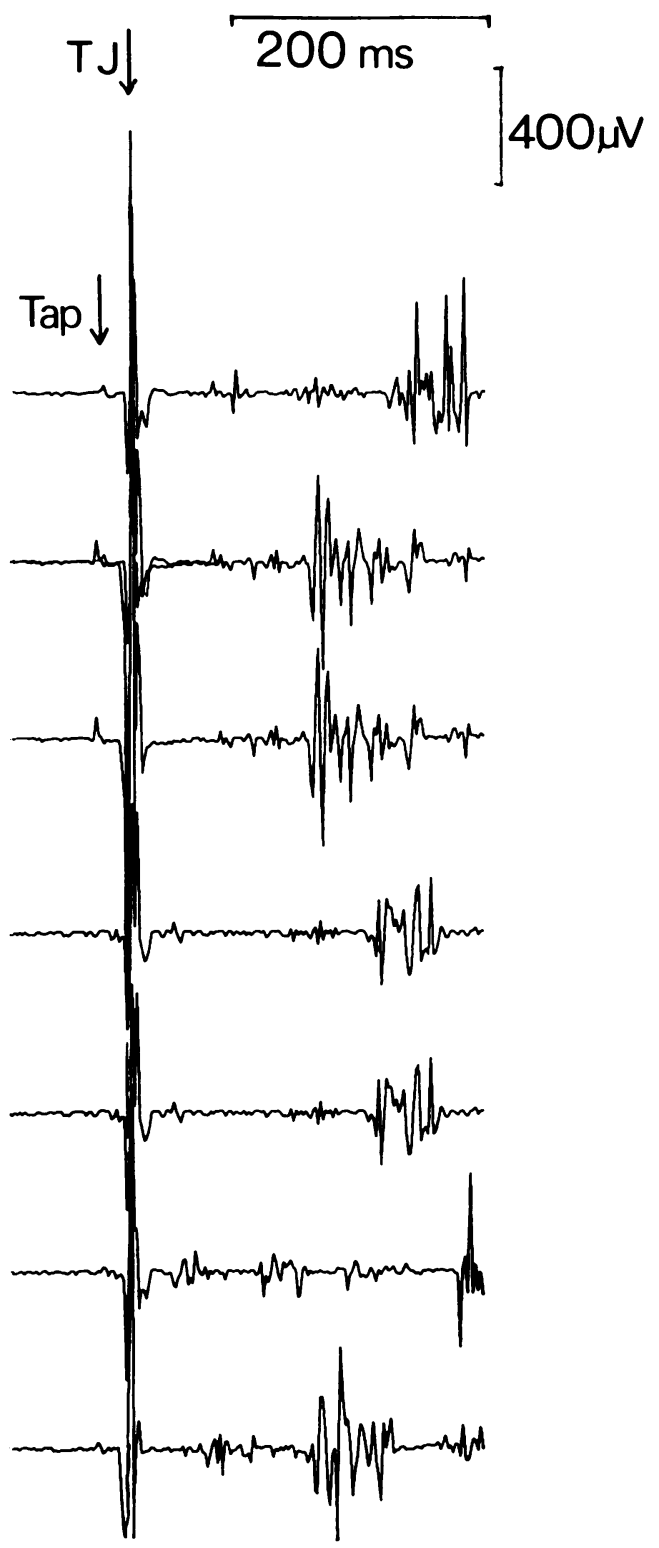

Fig. 4 EMG response of pronator teres to a tap with a tendon hammer (arrowed) as in Fig. 3. Seven consecutive single trials are shown. The tendon jerk response $(T J)$ in pronator teres at a fixed latency is followed by later responses with latencies varying from 128 to $232 \mathrm{~ms}$.

\section{EEG STUDIES}

The average evoked EEG recorded from the region of the sensorimotor cortex to taps on the right wrist is shown in Fig. 6. A large $(60 \mu \mathrm{V})$ slow positive potential appears bilaterally with 


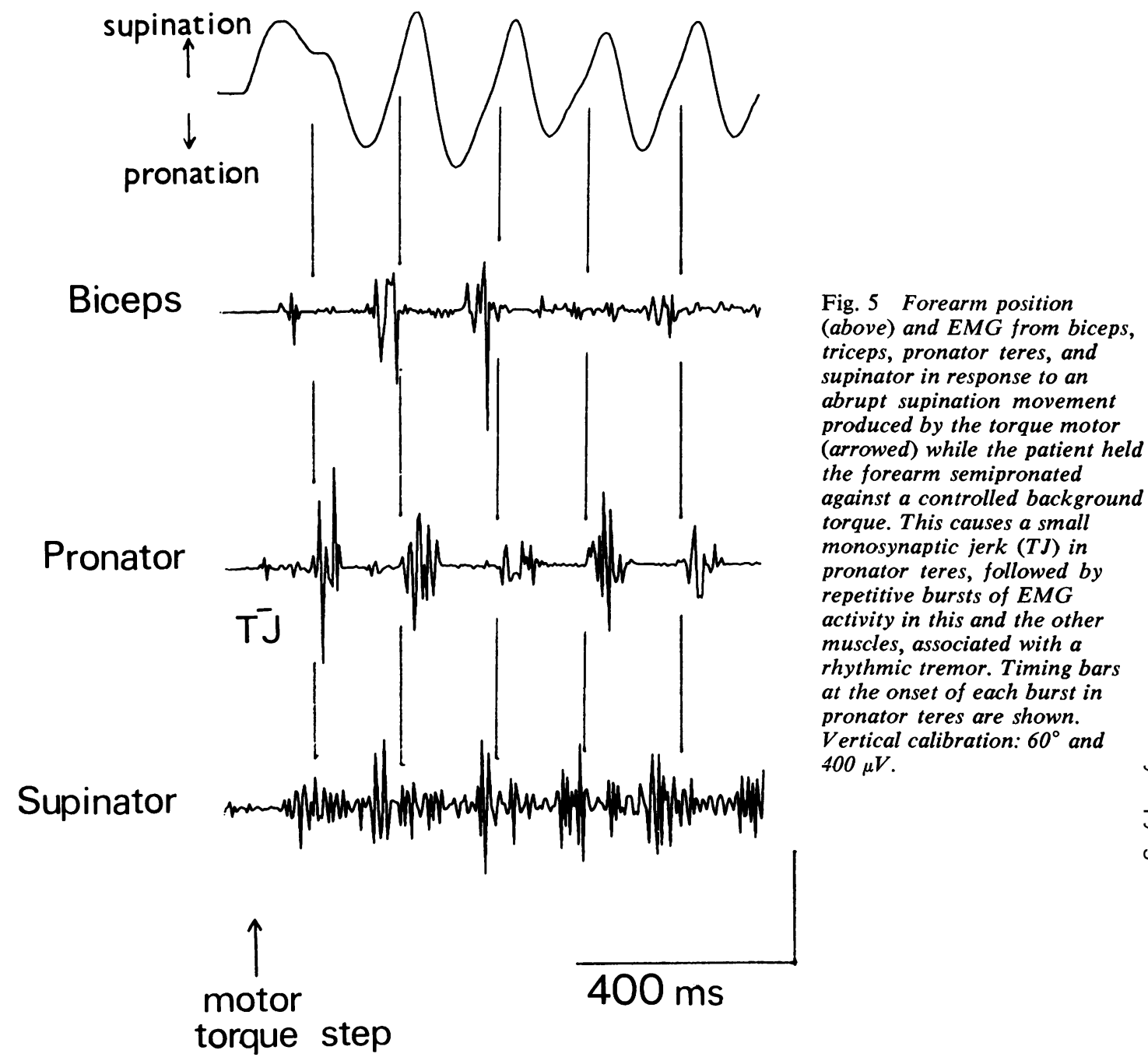

onset about $125 \mathrm{~ms}$ after the tap and with a peak at about $200 \mathrm{~ms}$ after the stimulus. The subsequent bursts of EMG activity were blurred in this averaged record because of variation in their latencies (jitter) relative to the tendon tap which triggered the averaging programme. However, EMG activity in biceps and supinator is evident, coincident with tremor beginning 55-60 ms after the tap. When the averaging was triggered by the first EMG burst of tremor in pronator teres, the EEG response was smaller but was time-locked to the EMG activity (Fig. 7). However, now the tendon jerk record was blurred because of jitter. The onset of the EEG positivity evoked by the tap preceded the EMG tremor burst and began at about the time of the monosynaptic tendon jerk. The cvoked EEG response was symmetrical over both hemispheres. There was no evidence of any localised left sided event to correlate with the tap to the right arm, even with more extensive recording sites 40 and $60 \mathrm{~mm}$ anterior and posterior to these positions. The EEG response to a jerk of the wrist by the torque motor so as to stretch pronator teres was similar (Fig. 8a).

\section{MOTOR POINT ANAESTHESIA}

On two occasions, $3 \mathrm{ml}$ of $1 \%$ lignocaine were injected into the motor point of the right pronator teres. Voluntary power was not reduced, but the abnormal tremor on writing or other pronation movements was abolished for four hours and the patient could write and draw with ease (Fig. 1c). 

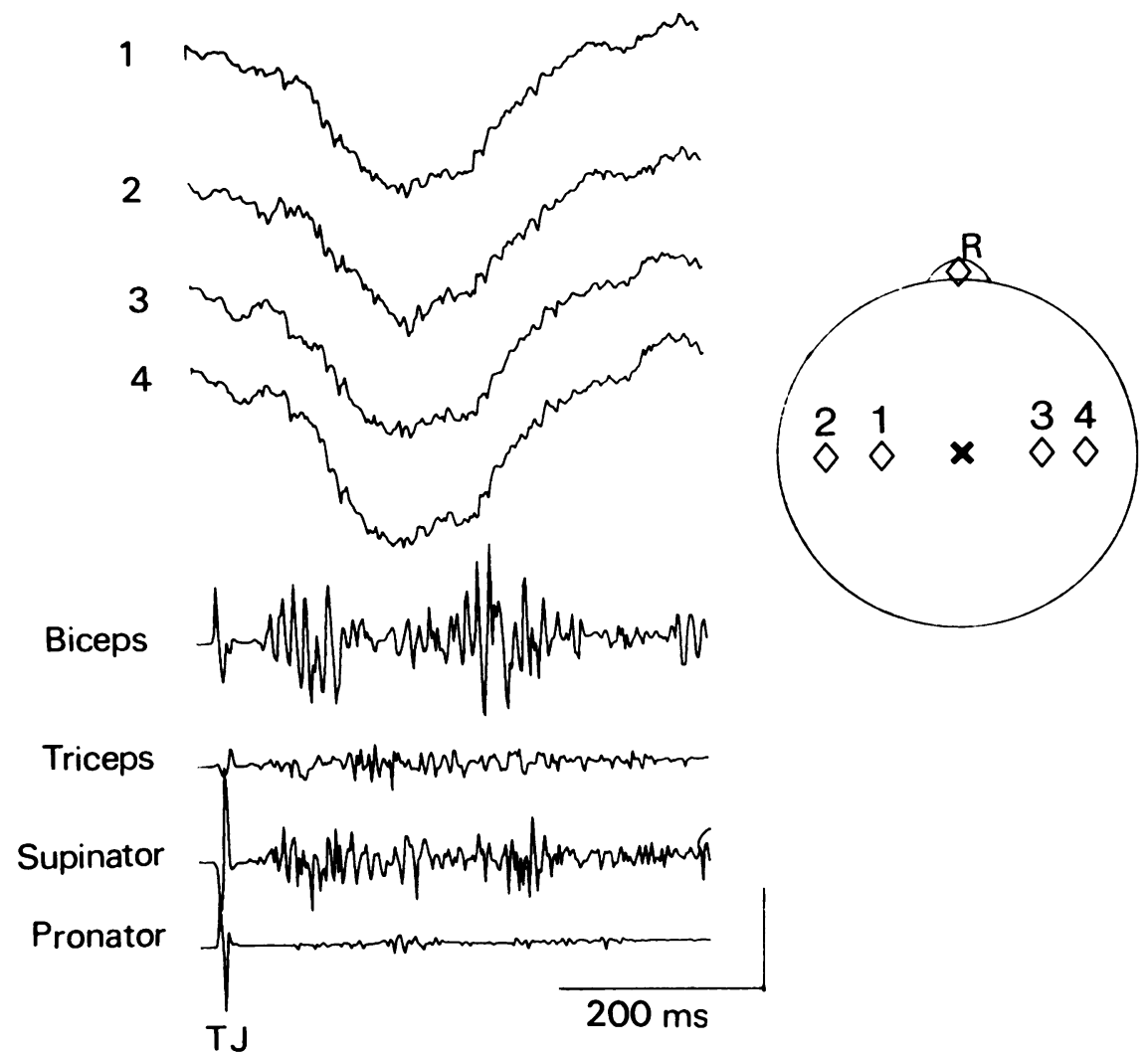

Fig. 6 Averaged EEG (above) and EMG responses from biceps, triceps, supinator, and pronator teres to 20 taps with a tendon hammer on the volar surface of the wrist. The sweeps were triggered by the tendon tap. The EEG montage is shown in the inset, and these unsmoothed records are with positivity downwards. The monosynaptic tendon jerk $(T J)$ shows little variation in latency, but the later bursts of tremor activity in biceps and supinator are ill-defined because of jitter in their latency with respect to the stimulus (see text). Vertical calibration: EMG $400 \mu V, E E G 40 \mu V$.

Tremor in response to a pronator stretch produced by the torque motor was also abolished, and the EEG responses evoked by this stimulus were reduced dramatically (Fig. 8b). Interestingly, tendon taps to pectoralis major could still elicit a small burst of tremor in biceps after anaesthesia of the motor point of pronator teres, although it was much reduced in amplitude compared to previous controls.

\section{Discussion}

This patient's complaint was that his arm jerked when he was writing. The jerks were so abrupt that they looked myoclonic, and at first sight his disability appeared to be a form of focal or segmental myoclonus. Segmental myoclonus has been described with a variety of lesions affecting the spinal cord (see Garcin et al., 1968; Halliday, 1975), but in such cases the jerking, which may be rhythmic, is spontaneous and persists for hours at a time and even in sleep. In addition, the frequency of jerking has been reported to be between 30 to 50 per minute and each burst of muscle action potentials has lasted a matter of a second or so. In all these respects our patient differs from those reported under the descriptive title of segmental myoclonus.

Myoclonic jerks on writing also occur in patients with torsion dystonia. Indeed, our patient's writing difficulty was somewhat reminiscent of dystonic writer's cramp in that the act of writing provoked the problem, as did other manual acts such as wielding a screwdriver or holding a cup. However, he exhibited no other dystonic features. The arm never adopted the 


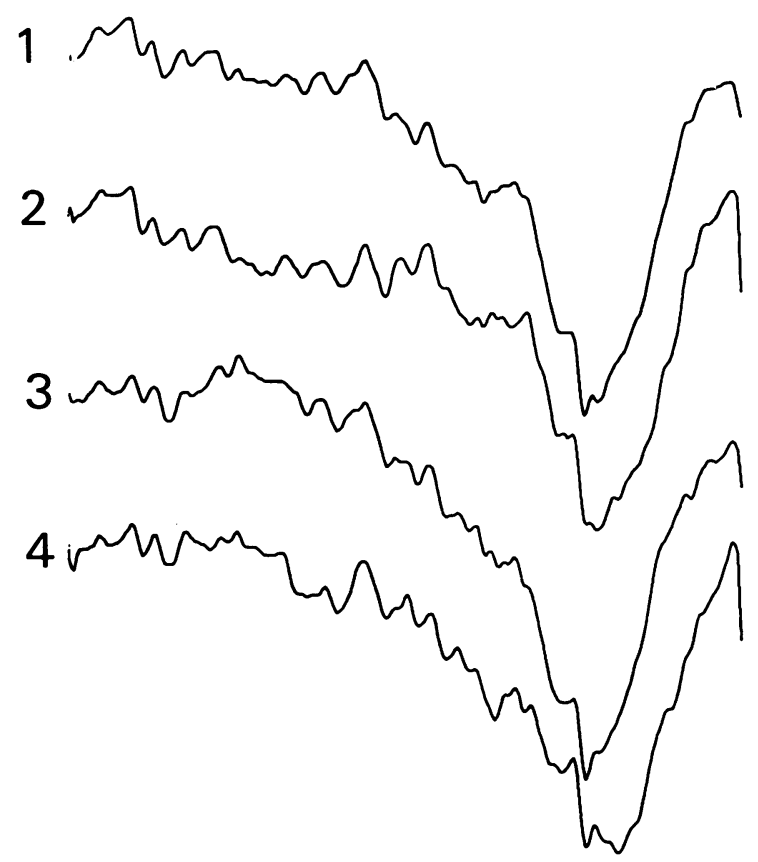

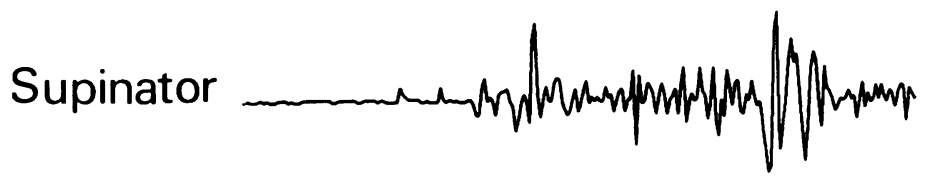

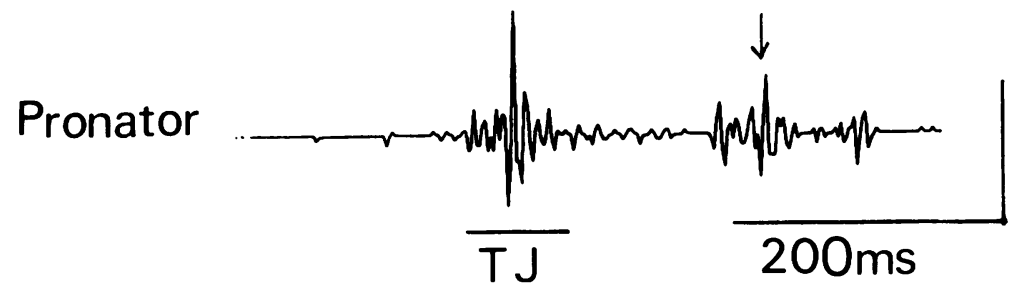

Fig. 7 Averaged $E E G$ (above, with montage as in Fig. 6) and EMG responses from supinator and pronator teres to 32 taps on the volar surface of the wrist with a tendon hammer as in Fig. 6. In this record the sweeps were triggered by the first burst of tremor in pronator teres (arrowed). The preceding tendon jerk response $(T J)$ now appears as a polyphasic burst of activity because of jitter in its latency with respect to the trigger point (see text). The EEG records have been smoothed using a digital 3 point moving average filter. Vertical calibration: EMG $400 \mu \mathrm{V}$; EEG $10 \mu \mathrm{V}$, positivity downwards. postures so characteristic of focal dystonia (elevation of the elbow and hyperpronation of the forearm or the forced grip of the fingers on the pen), and no sustained muscle spasms were witnessed. In all these respects his writing difficulty differed from that of dystonic writer's cramp.

Careful clinical and electrophysiological examination revealed that his "jerk" on writing was a short burst of tremor. Jerks also consisted of a burst of rhythmic oscillation of the arm. The correct description for his disability must be that of a focal tremor on particular action.

Action tremor is characteristic of benign essential tremor, which can occur unilaterally, certainly affects handwriting, and often occurs sporadically (Critchley M, 1949; Critchley E, 1972). Our patient exhibited other characteristics of benign essential tremor. Thus, his tremor was improved by alchohol at least temporarily, tremor frequency was about $6 \mathrm{~Hz}$ which is typical of that of benign essential tremor, and EMG studies indicated the presence of co-contraction of antagonistic muscles which also is frequently found in this condition, (Shahani and Young, 1978). However, in other respects our patient's disability differed markedly from that typically seen in benign essential tremor. Thus, he had no tremor of the outstretched hands or on attempting to hold his fingers in front of his nose. The tremor that did occur on pronation of his arm as in writing lasted only for a few beats, whereas benign essential tremor continues while the critical posture is held. Finally, his tremor 
(a)

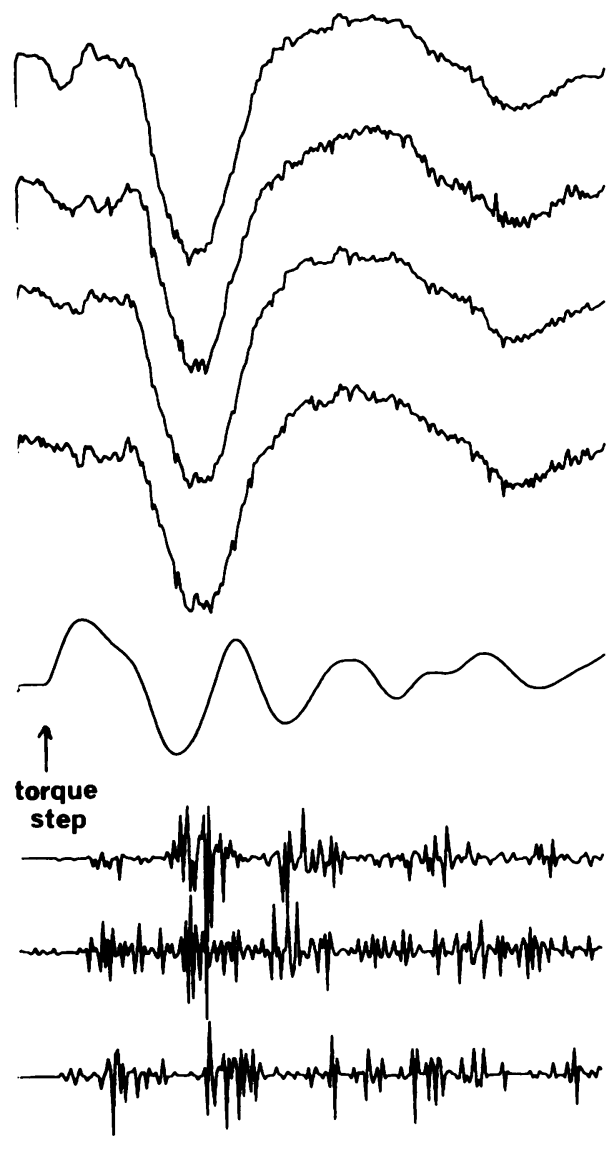

(b)
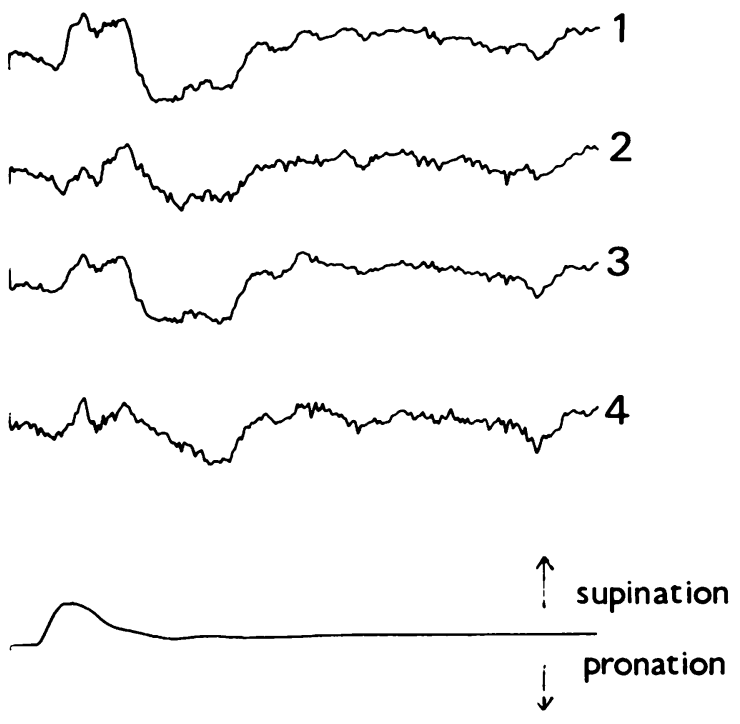

Biceps
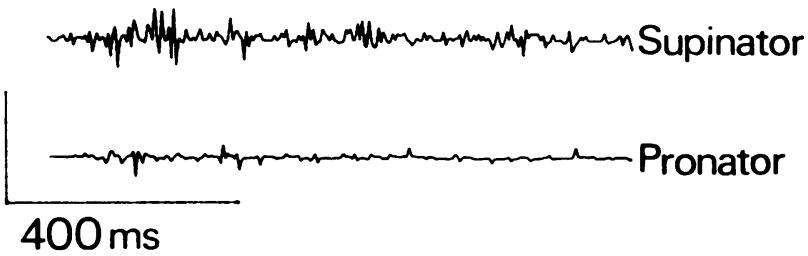

Fig. 8 Average EEG (upper four traces) (montage as in Fig. 6), forearm position, and EMG responses from biceps, supinator, and pronator teres (lower three traces) in response to forcible supination of the wrist by the torque motor (arrowed) while the patient held the forearm semipronated against a control background torque (32 trials). Vertical calibration: EEG $40 \mu V$, position $60^{\circ}, E M G 400 \mu V$. (a) Patient's usual response, (b) 15 minutes after infiltration of the motor point of pronator teres with $3 \mathrm{ml}$ of $1 \%$ lignocaine.

could be triggered by a tendon tap which is not conspicuous in benign essential tremor. We must conclude that our patient's tremor on writing was not that of typical benign essential tremor, although we cannot rule out the possibility that it represents an isolated variant of this condition.

Tremor on handwriting may occur as part of a more widespread and extensive neurological disorder such as dystonia, benign essential tremor, or Parkinson's disease, but none of these conditions was apparent in our patient. Therefore we would designate his disability as primary writing tremor.

The cause of this condition in our patient is obscure. It is tempting to relate it to his previous meningitis, but the long time interval between that illness and his writing difficulty, and the complete absence of any other evidence of neurological damage, make that suggestion unlikely. No other pathological cause is evident, and he is otherwise entirely well.

Likewise, the pathophysiology of the condition is uncertain, although our observations do allow some comments as to mechanisms. The important findings were that tendon taps delivered to supinate the forearm provoked a typical burst of tremor, and that this was subsequently abolished by partial motor point anaesthesia of pronator teres. This 
suggests that the critical stimulus was proprioceptive and, since the tendon jerk in pronator teres also was abolished by motor point anaesthesia, the critical stimulus appears to have been muscle spindle discharge. Voluntary activation of the same muscle (pronator teres) produced a similar tremor on writing and other such acts, and Vallbo (1974) has shown that muscle spindles fire faster during willed muscle contraction. Tremor during writing also was abolished by motor point anaesthesia at a time when there was no weakness, in keeping with the conclusion that it depended on muscle spindle activity.

Rondot et al. (1968) have also noted that motor point anaesthesia of a critical muscle (which they term the "indicator" muscle) may abolish postural tremor in benign essential tremor and cerebellar disease. They too argued that such tremor was generated by proprioceptive input from the critical muscle.

At what level in the nervous system might such proprioceptive input generate localised bursting tremor to cause this patient's difficulties? It might arise purely through spinal mechanisms, for although the latency of the first burst of tremor provoked by a tendon tap was very long it is now realised that long latency spinal events do occur (Ghez and Shinoda, 1978). Alternatively, the tremor might be generated by proprioceptive input acting in the brain. In this regard the presence of EEG events time-locked to the tremor bursts is of interest. However, these are unlikely to reflect a cortical origin of the tremor for two reasons. Firstly, the EEG events occurred in both hemispheres while the tremor was restricted to one arm. Secondly, the EEG events were not rhythmic and spanned two or three bursts of tremor (Fig. 8). Accordingly, it seems more likely that the changes recorded in the EEG were associated with the tremor, rather than responsible for it. The tremor might arise in brainstem, basal ganglia, or cerebellum, but we can provide no evidence to decide between these possibilities and it is fruitless to speculate further.

We are grateful to the Medical Research Council for financial support, and to $\mathrm{Mr} \mathrm{H}$. C. Bertoya and Dr B. L. Day for expert technical assistance.

\section{References}

Critchley, E. (1972). Clinical manifestations of essential tremor. Journal of Neurology, Neurosurgery, and Psychiatry, 35, 365-372.

Critchley, M. (1949). Observations on essential (heredofamilial) tremor. Brain, 71, 343-364.

Garcin, R., Rondot, P., and Guiot, G. (1968). Rhythmic myoclonus of the right arm as the presenting symptom of a cervical cord tumour. Brain, 91, 75-84.

Ghez, C., and Shinoda, Y. (1978). Spinal mechanisms of the functional stretch reflex. Experimental Brain Research, 32, 55-68.

Halliday, A. M. (1975). The neurophysiology of myoclonic jerking - a reappraisal. In Myoclonic Seizures (Excerpta Medica International Congress Series, No. 307), pp. 1-29. Edited by M. H. Charlton. Excerpta Medica: Amsterdam.

Marsden, C. D., and Parkes, J. D. (1973). Abnormal movement disorders. British Journal of Hospital Medicine, 10, 428-450.

Rondot, M. D., Korn, H., and Scherrer, J. (1968) Suppression of an entire limb tremor by anesthetising a selective muscular group. Archives of Neurology (Chicago), 19, 421-429.

Shahani, B. T., and Young, R. R. (1978). Action tremors: a clinical neurophysiological review. In Physiological Tremor, Pathological Tremors and Clonus, pp. 129-137. Edited by J. E. Desmedt. Karger: Basel.

Vallbo, A. B. (1974). Human muscle spindle discharge during isometric voluntary contractions. Amplitude relations between spindle frequency and torque. Acta Physiologica Scandinavica, 90, 319-336. 